\title{
Corrigendum
}

\section{Unfoldings in Knot Theory}

\author{
Walter Neumann ${ }^{1}$ and Lee Rudolph ${ }^{2}$ \\ ${ }^{1}$ University of Maryland, College Park, MD 20742, USA, and \\ Ohio State University, Columbus, OH 43210, USA \\ ${ }^{2}$ Clark University, Worcester, MA 01610, USA
}

In $[N-R]$ the link at infinity of a hypersurface $V \subset \mathbb{C}^{N}$ defined by a "good" polynomial map $f: \mathbb{C}^{N} \rightarrow \mathbb{C}$ was used as an extended example. $f$ was called "good" if it had only isolated singularities and it was claimed that the link at infinity then always has a "Milnor fibration." This is incorrect (although we have found it to be a common misconception). To correct it, the definition of "good" must be modified as follows.

Definition. The fiber $f^{-1}(c)$ of $f$ is regular ("ordinaire" in [S]) if there exists a neighborhood $D$ of $c$ in $\mathbb{C}$ such that $f \mid f^{-1}(D): f^{-1}(D) \rightarrow D$ is a locally trivial $C^{\infty}$ fibration and it is regular at infinity if there exists a neighborhood $D$ of $c$ in $\mathbb{C}$ and a compact set $K$ in $\mathbb{C}^{N}$ such that $f \mid f^{-1}(D)-K: f^{-1}(D)-K \rightarrow D$ is a locally trivial $C^{\infty}$ fibration. The polynomial map $f: \mathbb{C}^{N} \rightarrow \mathbb{C}$ is good if every fiber is regular at infinity. "Regular" is equivalent to "regular at infinity and non-singular." Whether $f$ is good or not, it has at most finitely many irregular fibers. We denote by $\mathscr{K}(f, \infty)$ the link at infinity of any fiber which is regular at infinity; up to isotopy this is independent of the choice of the fiber.

Example. $f(x, y)=x^{2} y+x$ is a polynomial with no singularities. The fiber $f^{-1}(0)$ is not regular at infinity since it has three components at infinity while nearby fibers only have two. $\mathscr{K}(f, \infty)$ is the 2-component link consisting of an unknot together with a $(2,-1)$ cable on it (so both components are unknotted and they have mutual linking 2); it is not a fiberable link.

With the above correction to terminology (synonyms to "good" - e.g. "having only isolated singularities" - must also be replaced), the results of $[\mathrm{N}-\mathrm{R}]$ remain correct, but the proof of 6.1 and the statement and proof of 7.1 need modification. From now on we assume ambient dimension $N=2$. The statements are:

6.1. Theorem (Converse to Milnor Fibration). If $N=2$ and $\mathscr{K}(f, \infty)$ is a fiberable link then $f$ is good.

7.1. Lemma (A Knot at Infinity is Good). If $V \subset \mathbb{C}^{2}$ is a fiber of $f: \mathbb{C}^{2} \rightarrow \mathbb{C}$ and is reduced and its link at infinity is a knot ( $V$ is connected at infinity) then $f$ is good. 
We first give a careful construction of the link at infinity, extracted from [N]. Call a manifold pair $(\Sigma, L)$ an abstract link at infinity for $\left(\mathbb{C}^{2}, V\right)$ if $(\Sigma, L) \times[0, \infty)$ is diffeomorphic to a neighborhood of infinity for the pair $\left(\mathbb{C}^{2}, V\right)$. Any two abstract links at infinity for $\left(\mathbb{C}^{2}, V\right)$ are diffeomorphic, since they are homotopy equivalent as pairs and one can therefore apply Waldhausen [W].

Let $n$ be the degree of $f$. By a linear change of coordinates $w=(x, y) \in \mathbb{C}^{2}$ we can put $f(x, y)$ in the form

$$
f(x, y)=x^{n}+f_{n-1}(y) x^{n-1}+\ldots+f_{0}(y) .
$$

Since $f$ has only finitely many irregular fibers, their images are all contained in the interior of some sufficiently large disk $D^{2}(s)=\{z \in \mathbb{C}|| z \mid \leqq s\}$ about the origin $0 \in \mathbb{C}$ Consider the polydisk $D(q, r)=\left\{(x, y) \in \mathbb{C}^{2}|| x|\leqq q| y \mid, \leqq r\right\}$.

Lemma. For $s$ as above sufficiently large, $r$ sufficiently large with respect to $s$, and $g$ sufficiently large with respect to $r$ and $s$, the fibers $f^{-1}(z)$ for $z \in \partial D^{2}(s)$ intersect $\partial D(q, r)$ only in the part $|x|<q,|y|=r$, and do so transversely - in fact, they intersect each line $y=y_{0}$ with $\left|y_{0}\right|=r$ transversely.

Proof. If, for given $r$ and $s, f^{-1}\left(D^{2}(s)\right)$ intersected $\{|x|=q,|y| \leqq r\}$ non-trivially for arbitrarily large $q$, then $y=0$ would be a point at infinity of the fibers $f^{-1}(z)$. This is not so, so for large $q, f^{-1}\left(D^{2}(s)\right)$ only meets the other part $\{|x|<q,|y|=r\}$ of $\partial D(q, r)$.

To see the transversality statement, consider $f(x, y)-z$ as a polynomial in $x$ with coefficients in $\mathbb{C}[y, z]$ and form its discriminant $\Delta \in \mathbb{C}[y, z]$ ( $\Delta$ is a polynomial in the coefficients of $f$ which vanishes if and only if $f=0$ has multiple roots). Then the fiber $f^{-1}\left(z_{0}\right)$ is transverse to the line $y=y_{0}$ if and only if $\Delta\left(y_{0}, z_{0}\right) \neq 0$. In particular, the fiber $f^{-1}\left(z_{0}\right)$ is regular at infinity if $\Delta(y, z) \neq 0$ for each $z$ close to $z_{0}$ and each $y$ of sufficiently large absolute value. But this fails if and only if $z=z_{0}$ is tangent to $\Delta(y, z)$ at infinity. In homogeneous coordinates $(y, z, w)$ at infinity, this says that $z=w=0$ is a point of $\Delta=0$ and $z=z_{0} w$ is a tangent line to $\Delta=0$ at this point. This can only happen for finitely many $z_{0}$, so we choose our disk $D^{2}(s)$ to contain these values in its interior.

Now choose $q, r$, and $s$, as in the above lemma. Let $D=f^{-1}\left(D^{2}(s)\right) \cap D(q, r)$. Its boundary is piecewise-smooth and decomposes as $\partial D=S \cup E$ with

$$
\begin{gathered}
S=\partial D(q, r) \cap f^{-1}\left(D^{2}(s)\right), \\
E=D(q, r) \cap \partial\left(f^{-1}\left(D^{2}(s)\right)\right) .
\end{gathered}
$$

$f$ restricts to a fibration of $E$ over a circle, and a typical fiber $F=f^{-1}(z) \cap E$ of $f \mid E$ satisfies: the pair $(\partial D, \partial F)$ is an abstract link at infinity for $\left(\mathbb{C}^{2}, V\right)$ (after smoothing the corner along $\partial S=\partial E)$. To see that $\left(\mathbb{C}^{2}-\operatorname{int}(D), f^{-1}(z)-\operatorname{int}(F)\right)$ is homeomorphic (diffeomorphic after smoothing corners) to $(\partial D, \partial F) \times[0, \infty)$ as desired, integrate along a suitable smooth vectorfield $v$ on $\mathbb{C}^{2}-\operatorname{int}(D)$ which is transversal inward on $\partial D$, is tangent to the fibers $f^{-1}(z)$ for $|y| \geqq r$ and $z \in \partial D^{2}(s)$, and whose $v$-derivative satisfies the following for some small $\varepsilon: v\left(|y|^{2}\right) \leqq-1$ when $|y| \geqq r-\varepsilon$ and $|f(x, y)| \leqq s+\varepsilon$, and $v\left(|f(x, y)|^{2}\right) \leqq-1$ otherwise. Such a vectorfield is easily constructed locally using the lemma, and a partition of unity then does it globally. 
If $f$ is good, we can choose $r$ sufficiently large that all fibers $f^{-1}(z)$ with $z \in D^{2}(s)$ are transverse to $|y|=r$. Then $S$ is equivalent to a disk tubular neighborhood of the link at infinity, so the construction has given the link at infinity with its Milnor fibration.

Proof of 6.1. Suppose $f$ is not good. The lemma implies that for $\left|y_{0}\right|=r$ the intersection $S_{0}=\left\{(x, y) \mid y=y_{0}\right\} \cap f^{-1}\left(D^{2}(s)\right)$ is transverse and $f \mid S_{0}: S_{0} \rightarrow D^{2}(s)$ is a holomorphic branched cover with no singularities over $\partial D^{2}(s)$. On the other hand it certainly does have singularities over int $D^{2}(s)$ (namely, near any fiber which is irregular at infinity). It follows that the inclusion $\partial F C S$ is not an isomorphism in homology. As in [N-R], this implies $\mathscr{K}(f, \infty)$ is not fiberable.

Proof of 7.1. By Suzuki [S], the general fiber of $f$ is also connected at infinity, so $\mathscr{K}(f, \infty)$ is a knot. As described in [N-R], it is an iterated torus knot, hence fiberable, so $f$ is good by Theorem 6.1.

\section{References}

[N-R] Neumann, W., Rudolph, L.: Unfoldings in knot theory. Math. Ann. 278, 409-439 (1987)

[N] Neumann, W.: Complex algebraic plane curves via their links at infinity. Preprint (1988)

[S] Suzuki, M.: Propriétés topologiques des polynômes de deux variables complexes, et automorphismes algébriques de l'espace $\mathbb{C}^{3}$. J. Math. Soc. Japan 26, 241-257 (1974)

[W] Waldhausen, F.: On irreducible 3-manifolds that are sufficiently large. Ann. Math. 87, 56-88 (1968)

Received April 7, 1988 